\title{
Modification of Liposomes by Addition of HCO60. II. Encapsulation of Doxorubicin into Liposomes Containing HCO60
}

\author{
Yasuki Kato, ${ }^{*}$ Toshihito Hosokawa, Yuichi Okubo, Eiji Hayakawa, and Kunio Ito \\ Pharmaceutical Research Laboratories, Kyowa Hakko Kogyo Co., Ltd., 1188, Shimotogari, Nagaizumi-cho, Sunto-gun, \\ Shizuoka 411, Japan. Received February 26, 1993
}

\begin{abstract}
The efficiency of encapsulation of doxorubicin (DOX) into liposomes containing HCO60 was studied. Empty egg phosphatidylcholine (PC) liposomes containing various amounts of $\mathrm{HCO60}$ were passed through a polycarbonate membrane filter $(0.1 \mu \mathrm{m})$. Closed vesicles were formed even with liposomes containing $90 \mathrm{wt} \%$ HCO60. DOX was encapsulated efficiently (more than $1 \mathrm{mg}$ DOX/8 mg lipids) using a pH-gradient method. HCO60 accelerated the leakage of DOX from liposomes into phosphate buffer at temperatures above $25^{\circ} \mathrm{C}$. $\mathrm{HCO60}$ suppressed the leakage of DOX from egg PC/cholesterol (7/3, w/w) liposomes into phosphate buffer containing $50 \%$ fetal bovine serum (FBS). Studies on the interaction between liposomes and serum components using zeta potential and turbidity suggest that HCO60 prevents the liposomes from interacting with serum components.
\end{abstract}

Keywords liposome; nonionic surfactant; doxorubicin; $\mathrm{pH}$ gradient; encapsulation

We have reported that the HCO60 micelle accumulates in the liver. ${ }^{1)}$ After obtaining these findings, we have been investigating the use of HCO60 for targeting drugs to the liver. Previously, we established that a liposome-bearing HCO60 was effective in targeting $\alpha$-tocopherol, chosen as a model hydrophobic drug, to the liver. ${ }^{2)}$ Liposomes are considered to be useful for the encapsulation of both water soluble and insoluble drugs. Doxorubicin (DOX) is an antitumor agent and many studies have been performed using liposomal DOX. The main reason for using liposomes is that they suppress cardiotoxic side-effects. ${ }^{3-5}$ Effects of liposomal DOX on liver metastases have been reported. ${ }^{6-8)}$ DOX can be encapsulated by many methods, e.g. using negatively charged lipids ${ }^{9-15)}$ or a $\mathrm{pH}$ gradient. ${ }^{16-21)}$ Encapsulation of DOX using a pH gradient is an effective method for obtaining a high encapsulation efficiency.

In the present report, we have studied some properties of liposomes bearing HCO60 and the encapsulation of DOX, a model water soluble drug, using a $\mathrm{pH}$ gradient. We also established a method of determining the interaction between liposomes and serum components by measuring of zeta potential. Law et al. ${ }^{22)}$ have reported an interaction between liposomes and bovine serum albumin (BSA) by measuring electrophoretic mobility. Mullar et $a l^{23)}$ and Troster et $a .^{24)}$ investigated the interaction between methyl-methacrylate nanoparticles and serum by measuring the zeta potential.

\section{Materials and Methods}

Materials Egg phosphatidylcholine (egg PC) was purchased from Nichiyu Liposome Ltd. Cholesterol was purchased from Nakarai Chemicals Ltd. HCO60 was purchased from Nikko Chemicals. Doxorubicin hydrochloride (DOX) was provided by Farmitalia Carlo Erba. Co. Potassium dihydrogenphosphate, disodium hydrogenphosphate, sodium carbonate, sodium hydroxide, and 2-propanol were purchased from Kanto Chemical Co. TritonX-100, ammonium molybdate, and citric acid were purchased from Wako Pure Chemical Industries Ltd. Fetal bovine serum (FBS) was purchased from Gibco Ltd. BSA was purchased from Sigma Ltd.

Preparation of Liposomes Multi-lamellar vesicles (MLV) were prepared by the method of Bangham et al ${ }^{25)}$ Egg PC or egg PC/cholesterol (egg $\mathrm{PC} / \mathrm{Chol}, 7 / 3, \mathrm{w} / \mathrm{w}$ ), and $\mathrm{HCO} 60$ were dissolved in $5 \mathrm{ml}$ 2-propanol (total amount of lipids and HCO60 was $0.5 \mathrm{~g}$ ), and the solvent was dried under vacuum in a glass test-tube. After addition of $5 \mathrm{ml} 50 \mathrm{~mm}$ citric acid solution, lipid films were hydrated by repeated vortex mixing. The MLV suspension was passed ten times through a polycarbonate membrane filter (Nucleopore, pore size $0.1 \mu \mathrm{m}$ Nomura Micro Science Ltd.) to size the liposomes by the method of Hope et al. ${ }^{26)}$ The mean particle size was determined using a dynamic laser light-scattering instrument (a model DLS-700, Otsuka Electronics Ltd.).

Encapsulation of DOX into Liposomes by pH Gradient Two mg DOX was dissolved in $1 \mathrm{ml}$ distilled water. Encapsulation of DOX was performed under two sets of conditions. The first was as follows: $100 \mu$ l DOX solution was added to $20 \mu \mathrm{l}$ liposome suspension, and then $80 \mu \mathrm{l} 100 \mathrm{~mm}$ sodium carbonate solution was added to the mixture. The $\mathrm{pH}$ gradient of this condition was 2.2-10.5. The second set of conditions was as follows: $100 \mu \mathrm{l}$ DOX solution was added to $20 \mu \mathrm{l}$ liposome suspension, and then $20 \mu \mathrm{l}$ water and $60 \mu 150 \mathrm{~mm}$ sodium carbonate were added to the mixture. The $\mathrm{pH}$ gradient under these conditions was 2.2-9.2. After mixing the DOX solution and liposome suspension, the mixture was incubated at 15 or $37^{\circ} \mathrm{C}$ for $10 \mathrm{~min}$.

Measurement of Encapsulation Efficiency of DOX into Liposomes The encapsulation efficiency of DOX into liposomes was measured as described by Mayer et al. ${ }^{20)}$ The DOX liposome was diluted with $25 \mathrm{~mm}$ pH 7.0 phosphate buffer (50-fold dilution). The absorbance at $600 \mathrm{~nm}$ of this diluted liposome suspension was adjusted to zero. $1 \mathrm{~N} \mathrm{NaOH}$ was added to the solution $(50 \mu 11 \mathrm{~N} \mathrm{NaOH} / 3 \mathrm{ml}$ of sample or $100 \mu 11 \mathrm{~N}$ $\mathrm{NaOH} / 3 \mathrm{ml}$ of FBS containing sample) to increase the $\mathrm{pH}$ (from 7 to 11). Immediately, the absorbance at $600 \mathrm{~nm}$ was measured (free DOX). The absorbance at $600 \mathrm{~nm}$ of $0.2 \%$ Triton X-100 solution was adjusted to zero. The absorbance at $600 \mathrm{~nm}$ of the liposomal DOX sample, after the addition of Triton X-100 (30 $\mu$ l of $20 \%$ Triton X-100/3 ml of sample), was measured (total DOX). The encapsulation efficiency (En. \%) was calculated according to the following equations;

En. $\%=(1-$ absorbance at $600 \mathrm{~nm}$ of free DOX/absorbance at $600 \mathrm{~nm}$ of total DOX) $\times 100$

Influence of BSA or FBS on the Zeta Potential of Empty Liposomes (without DOX) or HCO60 Micelles (without DOX) The liposome or HCO60 micelle suspension was diluted to give $0.5 \mathrm{mg}$ lipids including $\mathrm{HCO} 60 / \mathrm{ml}$ of $50 \mathrm{~mm}$ pH 7.0 phosphate buffer containing $1 \%$ BSA or $50 \%$ FBS. The solution was incubated at $37^{\circ} \mathrm{C}$ for $10 \mathrm{~min}$, and then the zeta potential of the solution was measured. The change of zeta potential ( $\Delta$ zeta potential) was obtained as follows;

$\Delta$ zeta potential $=($ zeta potential after the incubation with BSA or FBS $)$ -(initial zeta potential)

Initial zeta potential is shown in Table I.

Leakage of DOX from Liposome DOX liposomes were prepared as described above. DOX liposomes were diluted (50-fold dilution) with $25 \mathrm{~mm}$ pH 7.0 phosphate buffer or $50 \%$ FBS containing phosphate buffer. The diluted solution was incubated at $5-50^{\circ} \mathrm{C}$ for $30 \mathrm{~min}$. The encapsulation efficiency was immediately measured after the incubation, then the \% leakage of DOX, based on the initial encapsulation efficiency, was 
calculated.

Change in Turbidity in Liposome Suspension after Incubation with FBS Empty liposomes $(10 \mathrm{mg}$ lipids including $\mathrm{HCO} 60 / \mathrm{ml})$ were incubated with or without $5 \% \mathrm{FBS}$ at $37^{\circ} \mathrm{C}$ for $5 \mathrm{~min}$. The absorbance of the suspension at $450 \mathrm{~nm}$ was immediately measured after the incubation. The increase in turbidity in the liposome suspension without DOX, after incubation with $5 \%$ FBS, was obtained as a differential absorbance at $450 \mathrm{~nm}$ as follows;

(absorbance at $450 \mathrm{~nm}$ after incubation with FBS)

- (absorbance at $450 \mathrm{~nm}$ after incubation without FBS)

Measurement of Zeta Potential Zeta potentials were determined in $0.05 \mathrm{M} \mathrm{pH} 7$ phosphate buffer solution by electrophoretic measurements using a dynamic laser light-scattering detection electrophoretic apparatus (ELS-800, Otsuka Electronics Ltd.).

Electron Microscopy Negative-stain electron microscopy was performed according to the method of Magin and Weinstein. ${ }^{27)}$ Carboncoated copper grids (150-B mesh) were purchased from Ohken Shoji Ltd. A liposome drop was placed on the grid for 1 min and then removed. A drop of $2 \%$ ammonium molybdate was then placed on the grid and removed after $8 \mathrm{~min}$. The grid was then air dried and examined using an electron microscope (Hitachi $\mathrm{H}-7000,75 \mathrm{kV}$ of voltage).

\section{Results}

Physical Properties of HCO60 Liposomes Table I shows the physical properties of the HCO60 liposomes. The zeta potential of the liposomes increased slightly depending on the HCO60 content of the liposomes. The size of the liposomes containing $90 \%$ HCO60 was slightly smaller compared with liposomes containing less than $60 \%$ HCO60. Figure 1 shows the electron micrograms of liposomes using the nagative-staining method. Partial lipid lamellae were found in the liposomes containing $90 \%$

TABle I. Physical Properties of Egg PC Liposomes and Micelles

\begin{tabular}{ccc}
\hline HCO60 $(\%)$ & Size $(\mathrm{nm})$ & Zeta potential $(\mathrm{mV})$ \\
\hline 0 & $94.4 \pm 8.1$ & $1.15 \pm 1.15$ \\
10 & $97.5 \pm 11.5$ & $1.20 \pm 2.76$ \\
60 & $85.6 \pm 10.1$ & $2.65 \pm 2.22$ \\
90 & $77.3 \pm 8.1$ & $3.71 \pm 1.87$ \\
100 & $26.5 \pm 6.3$ & $5.26 \pm 1.12$
\end{tabular}

Data presented are the mean \pm S.D. of more than three experiments.
HCO60. In the $60 \%$ HCO60 liposomes, the repeat spacing of bilayers was slightly wider than that of the $100 \%$ PC liposomes.

Encapsulation Efficiency of DOX into Liposomes Figure 2 shows the encapsulation efficiencies of DOX into egg PC liposomes using the $\mathrm{pH}$ gradient method. Almost all the DOX was encapsulated into the $100 \%$ PC liposomes or $10 \%$ HCO60 liposomes. Encapsulation efficiency decreased when the liposome contained more than $60 \%$ HCO60.

Figure 3 shows the encapsulation efficiencies of DOX for the egg $\mathrm{PC} / \mathrm{Chol}(7 / 3, \mathrm{w} / \mathrm{w})$ liposomes using the $\mathrm{pH}$ gradient method. The encapsulation efficiency for the cholesterol-containing liposomes was lower than that for the egg PC liposomes at $15^{\circ} \mathrm{C}$. Encapsulation efficiencies increased with heating $\left(37^{\circ} \mathrm{C}\right)$.

Leakage of DOX from Liposomes into Phosphate Buffer DOX liposomes were examined without removing free DOX because the leakage study was performed under sink condition (50-fold dilution). Figure 4 shows the leakage of DOX from egg PC liposomes. Leakage of DOX increased with increasing the amount of HCO60. About $30 \%$ of the initial DOX encapsulated in the $60 \%$ HCO60 liposomes leaked at $37^{\circ} \mathrm{C}$ and almost all the DOX leaked at $50^{\circ} \mathrm{C}$. On the other hand, DOX did not leak from the liposomes composed of only egg PC. Figure 5 shows the leakage of DOX from the egg $\mathrm{PC} / \mathrm{Chol}$ liposomes.

Leakage of DOX from PC/Chol Liposomes into Phosphate Buffer Containing 50\% FBS Blood components are important factors involved in the leading of drug from liposomes. To obtain data on DOX leakage induced only by serum components, this test was performed at $15^{\circ} \mathrm{C}$ because no leakage of DOX was observed in phosphate buffer at $15{ }^{\circ} \mathrm{C}$. The leakage of DOX from liposomes into the buffer containing 50\% FBS was examined. Figure 6 shows the leakage of DOX from liposomes at $15^{\circ} \mathrm{C}$. HCO60 suppressed the leakage of DOX from liposomes into $50 \%$ FBS buffer.

Influence of BSA or FBS on the Zeta Potential of Liposomes or HCO60 Micelles The zeta potential of lipo-
A

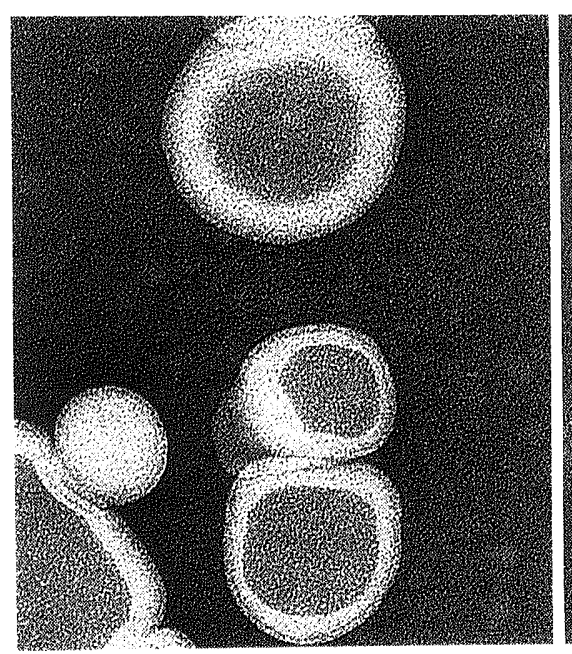

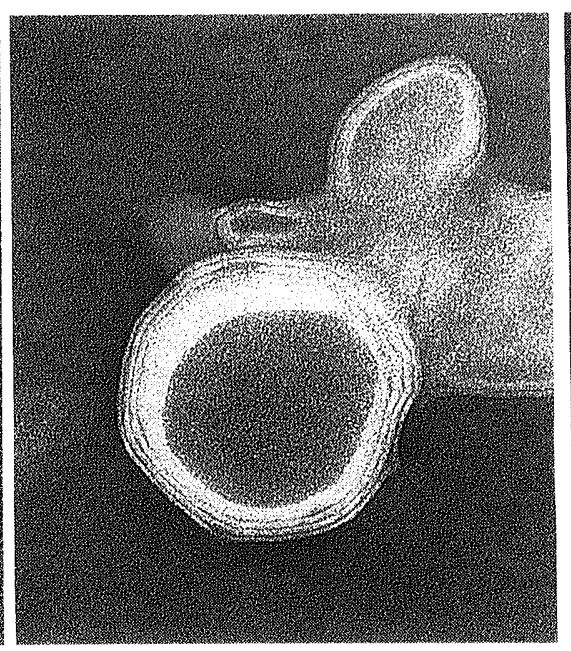

C

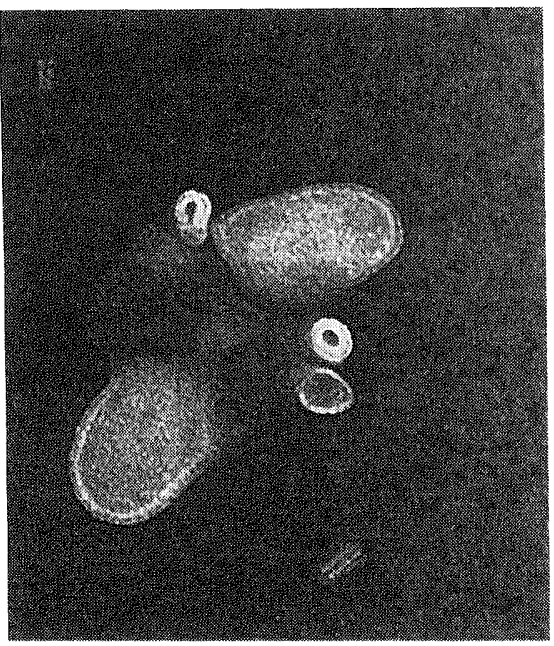

Fig. 1. Electron Microscope Images of Liposomes Containing Various Amounts of HCO60 by Negative Staining Magnification, $\times 100000$; size of bar, $100 \mathrm{~nm}$. A, 100\% PC; B, 60\% HCO60; C, $90 \%$ HCO60. 


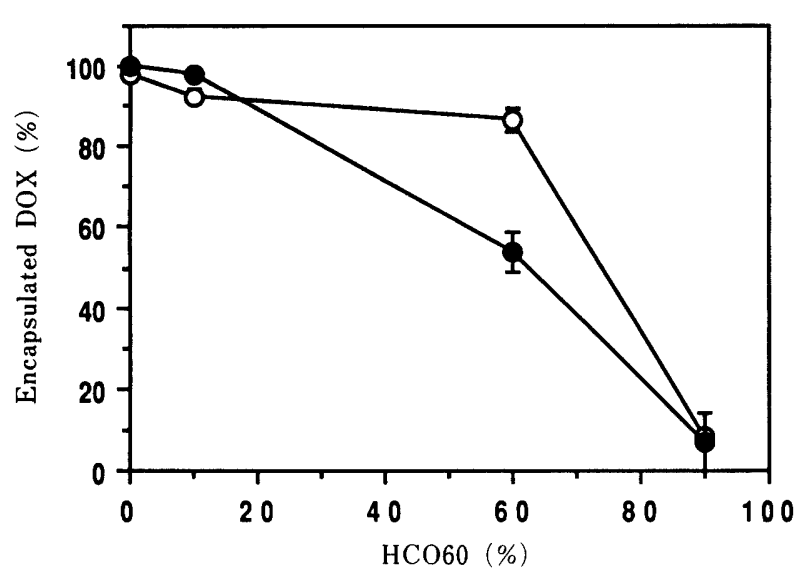

Fig. 2. Encapsulation of DOX into Egg PC Liposomes by the pH Gradient Method

Data presented are the mean \pm S.D. of three experiments. $O$, pH $2.2 \rightarrow 10.5$ at $15^{\circ} \mathrm{C} ; 0, \mathrm{pH} 2.2 \rightarrow 10.5$ at $37^{\circ} \mathrm{C}$.

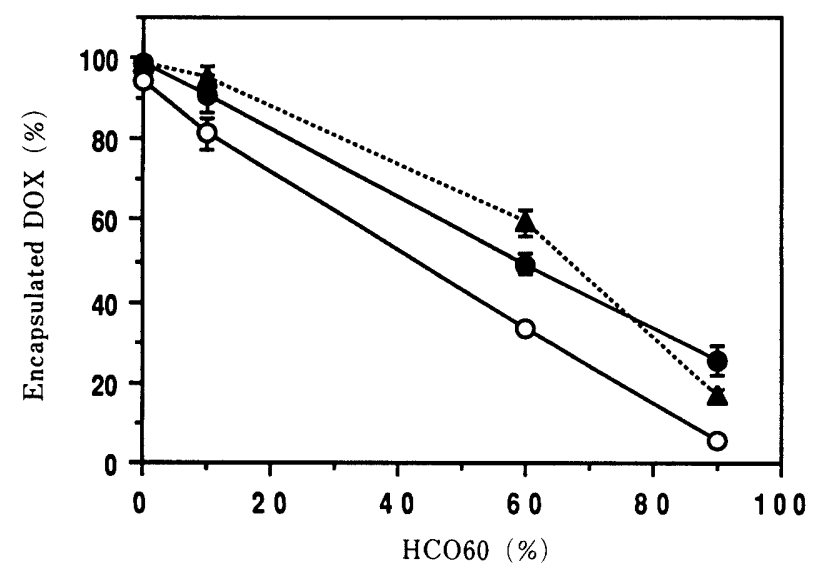

Fig. 3. Encapsulation of DOX into Egg $\mathrm{PC} / \mathrm{Chol}(7 / 3, \mathrm{w} / \mathrm{w})$ Liposomes by the $\mathrm{pH}$ Gradient Method

Data presented are the mean \pm S.D. of three experiments. $O$, $\mathrm{pH} 2.2 \rightarrow 10.5$ at $15^{\circ} \mathrm{C} ; \boldsymbol{\rho}, \mathrm{pH} 2.2 \rightarrow 10.5$ at $37^{\circ} \mathrm{C} ; \boldsymbol{\Delta}, \mathrm{pH} 2.2 \rightarrow 9.2$ at $37^{\circ} \mathrm{C}$.

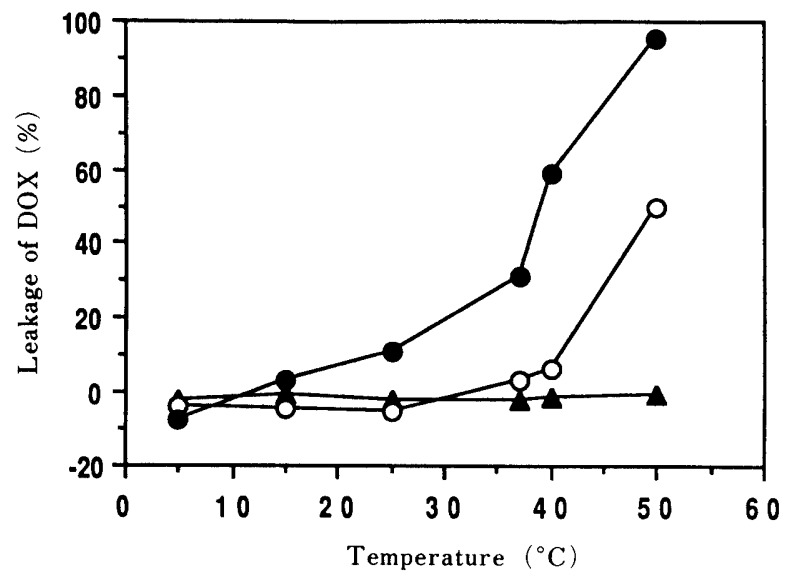

Fig. 4. Leakage of DOX from Egg PC Liposomes after Incubation in Phosphate Buffer for $30 \mathrm{~min}$

A, $100 \%$ PC; O, $10 \%$ HCO60;, $60 \%$ HCO60

somes or HCO60 micelles without DOX after incubation with $1 \%$ BSA or $50 \%$ FBS in $50 \mathrm{~mm} \mathrm{pH} 7.0$ phosphate buffer at $37^{\circ} \mathrm{C}$ for $10 \mathrm{~min}$ was measured. Figure 7 shows the change in zeta potential ( $\Delta$ zeta potential). In the case of BSA, the $\Delta$ zeta potential of liposomes without HCO60

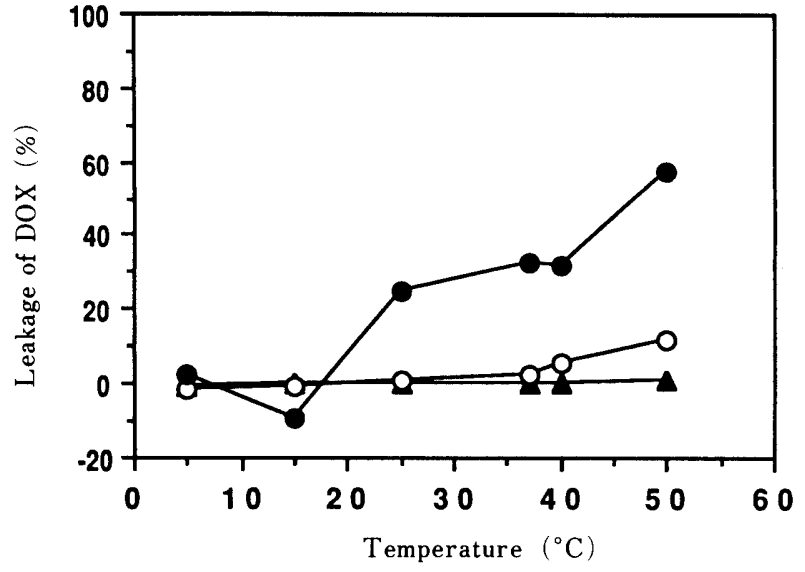

Fig. 5. Leakage of DOX from Egg PC/Chol (7/3, w/w) Liposomes after Incubation in Phosphate Buffer for $30 \mathrm{~min}$

A, $100 \%$ PC; O, $10 \%$ HCO60;, $60 \%$ HCO 60 .

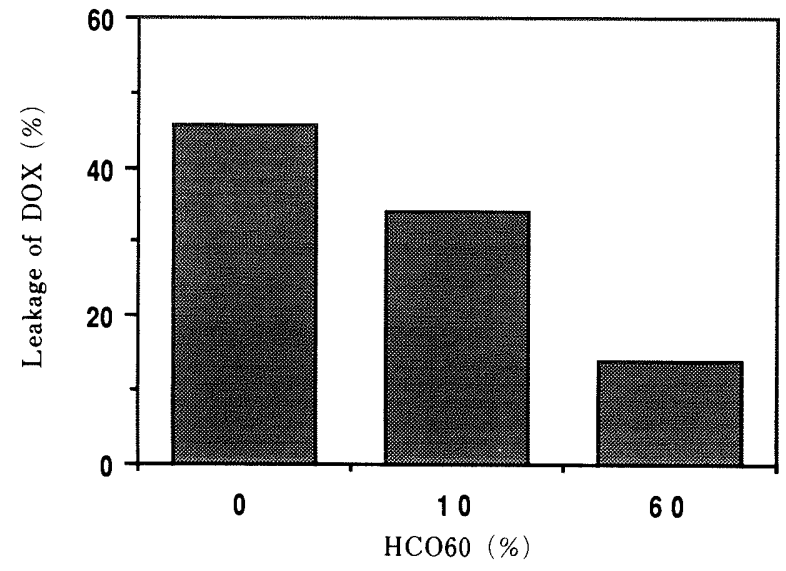

Fig. 6. Leakage of DOX from Egg PC/Chol (7/3, w/w) Liposomes after Incubation in Phosphate Buffer Containing $50 \%$ FBS at $15^{\circ} \mathrm{C}$ for $30 \mathrm{~min}$

was $-8 \mathrm{mV}$ and the addition of $10 \% \mathrm{HCO} 60$ to liposomes reduced the change of zeta potential $(-6 \mathrm{mV})$. However, the $\Delta$ zeta potential of liposomes containing more than $60 \%$ HCO60 was similar to that of HCO60 micelles. In the case of FBS, the $\Delta$ zeta potential of liposomes with $0 \%, 10 \%, 60 \%$, and $90 \%$ HCO60 was +2.4 , $+0.6,-1.9$ and $-1.2 \mathrm{mV}$, respectively.

Change in Turbidity of Liposome Suspensions after Incubation with FBS The increase in turbidity in the liposome suspension without DOX after incubation with $5 \%$ FBS was measured. HCO60 suppressed the increase in turbidity in the liposome suspension by FBS, as shown in Fig. 8.

\section{Discussion}

The closed vesicle composed of lipids and HCO60 was obtained even in the case of $60 \% \mathrm{HCO} 60$ and partial lipid lamellae were found in liposomes containing 90\% HCO60 (Fig. 1). This suggests that HCO60 can form a liposome with phospholipids even if a high content of HCO60 is used. The influence of HCO60 on the repeat spacing of the lipid bilayer was examined by electron microscopy. The repeat spacing of dipalmitoylphosphatidylcholine (DPPC) liposomes has been reported to be enlarged by the addition of Triton (polyoxyethylene (POE)-nonyl-phenyl alkyl), and 


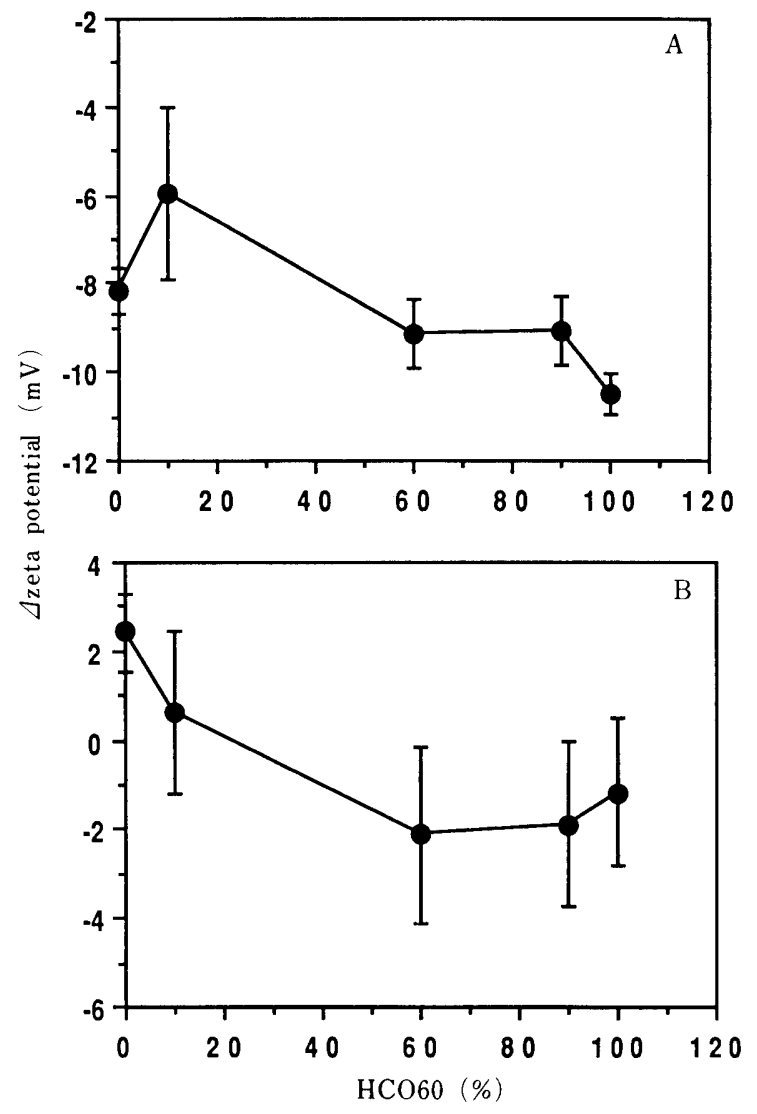

Fig. 7. Change of Zeta Potential of Liposomes Containing Various Amounts of $\mathrm{HCO} 60$ or $\mathrm{HCO} 60$ Micelles after Incubation at $37^{\circ} \mathrm{C}$ for $10 \mathrm{~min}$

Data presented are the mean + S.D. of three experiments. A, incubation with $1 \%$ BSA; $B$, incubation with $50 \%$ FBS

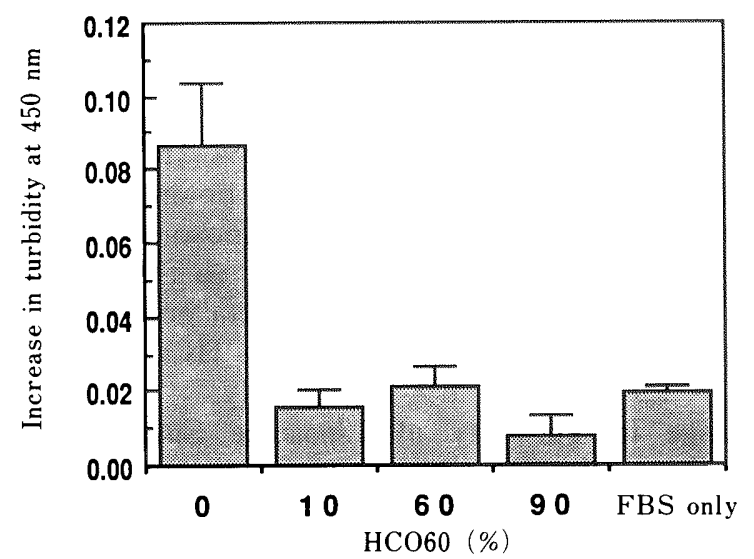

Fig. 8. Change of Turbidity in Liposome Suspension after Incubation with $5 \%$ FBS Solution at $37^{\circ} \mathrm{C}$ for $5 \mathrm{~min}$

Data presented are the mean \pm S.D. of three experiments.

the surfactant having 10 POE groups has a space about $2 \mathrm{~nm}$ wider over the phase transition temperature. ${ }^{28)}$ Saito et al. ${ }^{29)}$ reported that $\mathrm{HCO} 60$ had $1.71 \mathrm{~g}$ of water bound per $1 \mathrm{~g}$ of HCO60. We speculate that the bound water of HCO60 is important in widening the lipid bilayer space of the liposomes.

Encapsulation of DOX into liposomes was performed by the $\mathrm{pH}$ gradient method. In the case of egg PC liposomes, the encapsulation efficiency of DOX in the $60 \% \mathrm{HCO} 60$ liposomes was lower than that of liposomes containing less than $10 \%$ HCO60. Nevertheless, $1 \mathrm{mg}$ DOX per $8 \mathrm{mg}$ phospholipid was encapsulated into liposomes by $\mathrm{pH}$ $2.2 \rightarrow 10.5$. This level can be considered to be high compared with the method using negatively charged lipid for encapsulation of DOX into liposomes. On the other hand, encapsulation of DOX was suppressed by the addition of cholesterol to egg $\mathrm{PC}$ at $15^{\circ} \mathrm{C}$. This was solved by modification of the conditions, i.e. temperatue and $\mathrm{pH}$, used in the encapsulation process for DOX. These findings suggest that the liposomal bilayer formed with lipids and HCO60 is a closed vesicle and can be called a liposome. Maruyama et al. ${ }^{30)}$ reported that $95 \%$ of the DOX was encapsulated into liposomes bearing $6 \mathrm{~mol} \%$ ganglioside GM1 using a $\mathrm{pH}$ gradient method. Similar encapsulation efficiency was obtained in the case of $10 \mathrm{wt} \%$ (approximately $2 \mathrm{~mol} \%$ ) HCO60 liposomes. Addition of HCO60 induced leakage of DOX from liposomes into phosphate buffer, depending on the amount of HCO60 added to the liposomes. Addition of cholesterol was effective in suppressing the leakage of DOX from HCO60-containing liposomes at $50^{\circ} \mathrm{C}$. Nevertheless, the leakage of DOX from egg $\mathrm{PC} / \mathrm{Chol}$, containing $60 \% \mathrm{HCO} 60$, was greater than that from egg PC containing $60 \% \mathrm{HCO} 60$ at $25^{\circ} \mathrm{C}$. We speculate that this unexpected result may be caused by the phase separation of cholesterol in the membrene, induced by the addition of $\mathrm{HCO} 60$. Arnold et al. ${ }^{28)}$ reported that the phase transition temperature of DPPC was increased fron 41 to $45^{\circ} \mathrm{C}$ by the addition of Triton having a long POE chain (30 POE). These characteristics suggest that HCO60 affects the fluidity of the lipid membrane. In this respect, HCO60 is similar to cholesterol. It is well known that a certain amount of cholesterol relative to phospholipids induces phase separation of lipids in the lipid bilayer and the temperature of this phase separation change depends on the ratio of cholesterol to phospholipids. ${ }^{31)}$ HCO60 may have affected the phase separation characteristics in the $\mathrm{PC} / \mathrm{Chol}$ lipid membrane. HCO60 suppressed leakage of DOX from the liposomes to $50 \%$ FBS buffer. The leakage of water-soluble drugs is accelerated by serum components such as high density lipoprotein (HDL). ${ }^{32)} \mathrm{HCO} 60$ can prevent liposomes from interacting with blood components.

This protective effect of HCO60 was demonstrated by measuring the zeta potential of the liposomes. BSA has a negative charge in a solution of neutral $\mathrm{pH}$, because the isoelectric point of BSA is $4.7 .^{33)}$ Since the zeta potential of liposomes was shifted to a negative value after incubation with BSA (Fig. 7A), this suggests that BSA bind to the liposome. HCO60 (10\%) slightly suppressed the binding of BSA onto the liposomes. BSA binding may have occurred due to an electrostatic interaction between BSA and liposomes or micelles. On the other hand, HCO60 suppressed the positive shift in the zeta potential of liposomes induced by FBS (Fig. 7B). Positively-charged components having an isoelectric point higher than 7 , such as $\mathrm{ApoB},{ }^{34)} \mathrm{ApoC}-\mathrm{I},{ }^{35)} \mathrm{IgM},{ }^{36)}$ and plasminogen, ${ }^{36)}$ may bind liposomes without HCO60. HCO60 may prevent binding between liposomes and hydrophobic substances, such as ApoB or ApoC-I, in these components. HCO60 may render the liposome surface hydrophilic because HCO60 has a large amount of bound water $(1.17 \mathrm{~g} / \mathrm{g}$ micelle $\left.{ }^{29}\right)$, and then hydrophobic components such as 
ApoB or ApoC-I will not be able to bind to the liposomes. These studies were performed using liposomes without DOX. It is believed that DOX exists in the inner space of the liposome, after the encapsulation using the $\mathrm{pH}$ gradient method, so the zeta potential of the liposomes might not be affected by DOX encapsulated in the liposome.

After experimenting with conditions to obtain a clear change in turbidity of the liposomes produced by FBS, the concentration of FBS was chosen as $5 \%$. Increase in turbidity might be induced by binding of serum components. HCO60 was quite effective in preventing the binding of serum components to liposomes (Fig. 8). Even in the case of $10 \%$ HCO60 liposomes, this effect was still observed. It is possible that HCO60 could easily prevent the binding of serum proteins because the concentration of FBS in this experiment was not high. This finding suggests that HCO60 suppresses the binding of serum components inducing the leakage of DOX from the liposomes.

We have demonstrated that HCO60 liposomes are suitable for the encapsulation of hydrophobic compounds such as $\alpha$-tocopherol (preceding paper) and liposomes containing more than $60 \%$ HCO60 are accumulated in the liver. Allen et al. ${ }^{37)}$ and Liu et al. ${ }^{38)}$ reported that the accumulation of ganglioside GM1-bearing liposomes with a diameter of approximately $50 \mathrm{~nm}$, in the liver was greater than that of larger liposomes and they speculated that these liposomes were taken up by liver parenchymal cells. A similar phenomenon was observed in the case of polyethylene glycol (PEG)-modified liposomes. ${ }^{39)}$ Liposomes with a large amount of PEG-lipids have been considered to induce fusion with cells. ${ }^{40)}$ Liposomes containing a large amount of HCO60 may also induce fusion with liver cells. Ganglioside GM1 liposomes seem to be taken up through endocytosis by liver parenchymal cells and this is the difference between them and HCO60 liposomes. This fusion-inducing property seems to be an advantage in clinical treatment using liposomal drugs, because the liposomes encapsulating the drug are degraded in the lysosome after endocytosis by the cells. Of course, further experiments are needed to fully describe this phenomenon. In conclusion, the HCO60 liposome is a useful carrier for the delivery of water-soluble and -insoluble drugs to the liver.

Acknowledgement We are very grateful to Mr. Harumi Mochizuki and Ms. Hiroko Yoshitake for their technical assistance with the electron microscopy, and to Mr. Hirotake Naganuma, Yuji Kawaguchi and Ms. Hiroe Katsumata, for their valuable technical assistance.

\footnotetext{
References

1) Y. Kato, K. Watanabe, M. Nakakura, T. Hosokawa, E. Hayakawa, K. Ito, Chem. Pharm. Bull., 41, 599 (1993).

2) Y. Kato, K. Watanabe, T. Hosokawa, E. Hayakawa, K. Ito, Biol. Pharm. Bull., 16, 960 (1993).

3) E. A. Forssen, Z. A. Tokes, Proc. Natl. Acad. Sci. U.S.A., 78, 1873 (1981).

4) A. Gabizon, A. Neshore, Y. Barenholz, J. Natl. Cancer Inst., 77, 459 (1986).
}

5) J. A. E. Balazsovits, L. D. Mayer, M. B. Bally, P. R. Cullis, M. McDonell, R. S. Ginsberg, R. E. Falk, Cancer Chemother. Pharmacol., 23, 81 (1984).

6) A. Gabizon, D. Goren, Z. Fuks, Y. Barenholz, A. Dagan, A. Meshorer, Cancer Res., 43, 4730 (1983).

7) A. Gabizon, D. Goren, Z. Fuks, A. Meshorer, Y. Barenholz, Br. J. Cancer, 51, 681 (1985).

8) E. G. Mayhew, M. H. Goldrosen, J. Vaage, Y. M. Rustum, J. Natl. Cancer Inst., 78, 707 (1987).

9) R. L. Juliano, D. Stamp, Biochem. Pharmacol., 27, 21 (1978).

10) E. A. Forssen, Z. A. Tokes, Biochem. Biophys. Res. Commun., 91, 1295 (1979).

11) A. Rahman, A. Kessel, N. More, B. Sikic, G. Rowden, P. Wooley, P. S. Schein, Cancer Res., 40, 1532 (1980).

12) A. Gabizon, A. Dagan, D. Goren, Y. Barenholz, Z. Fuks, Cancer Res., 42, 4734 (1982).

13) F. Olson, E. Nayhew, D. Maslow, T. Rustum, F. Szoka, Eur. J. Cancer Clin. Oncol., 18, 471 (1986).

14) P. Rosa, F. Clementi, Pharmacology, 221 (1983).

15) N. Kojima, N. Ueno, M. Takano, H. Yabushita, M. Noguchi, M. Ishihara, K. Yagi, Biotechnology and Applied Biochemistry, 8, 471 (1986).

16) L. D. Mayer, M. B. Bally, M. J. Hope, P. R. Cullis, Biochim. Biophys. Acta, 816, 294 (1985).

17) L. D. Mayer, M. J. Hope, P. R. Cullis, A. S. Janoff, Biochim. Biophys. Acta, 817, 193 (1985).

18) L. D. Mayer, M. B. Bally, P. R. Cullis, Biochim. Biophys. Acta, 857, 123 (1986).

19) L. D. Mayer, M. B. Bally, M. J. Hope, P. R. Cullis, Chem. Phys. Lipids, 40, 333 (1986).

20) L. D. Mayer, L. C. L. Tai, M. B. Bally, G. N. Mitilenes, R. S. Ginsberg, P. R. Cullis, Biochim. Biophys. Acta, 1025, 143 (1990).

21) E. Hayakawa, M. Nakakura, Y. Kato, Y. Okubo, T. Hosokawa, Chem. Pharm. Bull., 39, 773 (1991).

22) S. L. Law, W. Y. Lo, S. H. Pai, G. W. Teh, Int. J. Pharmaceut., 43, 257 (1988).

23) R. H. Muller, K. H. Wallins, S. D. Troster, J. Kreuter, J. Controlled Release, 20, 237 (1992).

24) S. D. Troster, K. H. Wallins, R. H. Muller, J. Kreuter, J. Controlled Release, 20, 247 (1992).

25) A. D. Bangham, M. M. Atandish, J. C. Watkins, J. Mol. Biol., 13, 238 (1965).

26) M. J. Hope, M. B. Bally, G. Webb, P. R. Cullis, Biochim. Biophys. Acta, 812, 55 (1985).

27) R. L. Magin, J. N. Weinstein, "Liposome Technology," Vol. III, ed. by G. Gregoriadis, CRC Press Inc., Florida, 1984, p. 142.

28) K. Arnold, Y. M. Lvov, M. Szogyi, S. Gyorgri, Studia Biophysica, 113, 7 (1986).

29) Y. Saito, T. Sato, Yakugaku Zasshi, 112, 763 (1992).

30) K. Maruyama, A. Okamoto, S. Kojima, A. Suginaka, M. Iwaturu, Drug Deliv. System, 7, 345 (1992).

31) T. Handa, "Makugaku Nyumon," ed. by M. Nakagaki, Kitami Shoboh, Tokyo, 1978, pp. 89--106.

32) G. Scherphof, H. Morselt, Biochem. J., 221, 423 (1984).

33) "Seikagaku Jiten," Tokyo Kagaku Dohjin, Tokyo, 1984, p. 411.

34) B. C. Melnik, S. F. Melnik, Anal. Biochim., 171, 320 (1988).

35) M. J. Chapman, Methods Enzymol., 128, 70 (1986).

36) "Seikagaku Data Book I," Tokyo Kagaku Dohjin, Tokyo, 1979, pp. $129-135$.

37) T. M. Allen, C. Hansen, J. Rutledge, Biochim. Biophys. Acta, 981, 27 (1989).

38) D. Liu, A. Mori, L. Huang, Biochim. Biophys. Acta, 1104, 95 (1989).

39) T. M. Allen, C. Hansen, F. Martin, A. Yau-Young, Biochim. Biophys. Acta, 1066, 29 (1991).

40) M. C. Woodle, D. D. Lasic, Biochim. Biophys. Acta, 1113, 171 (1992). 